\title{
Generalization of the Jared-Ennis method to complex transmittance objects for the generation of Synthetic Discriminant Function Filters
}

\author{
Encarnación Pleguezuelos, Ignasi Labastida, Santiago Vallmitjana and Artur Carnicer \\ Departament de Física Aplicada i Òptica. Universitat de Barcelona. \\ Avda. Diagonal 647, E08028 Barcelona, Spain
}

\begin{abstract}
In this work we present a generalization to complex transmittance objects of the Jared-Ennis algorithm for the generation of Synthetic Discriminant Function filters (SDFs). The original algorithm consists of the resolution of a nonlinear system of equations by means of an iterative procedure, including a phase adaptation of the filter. The method shown here takes into account the modulation of liquid crystal displays (LCD) both for scene and filter, generalizing the problem to the complex plane. Considering this new method gives a more realistic picture as the LCD modulation gives a complex distribution of the scenes instead of only real values as considered before. For instance, we use a high contrast configuration to display the scenes. Moreover, the addition of new parameters to the problem allows us to consider filters other than the phase-only one. In our case, we use a phase-mostly configuration to display the filter and the metric optimized is the maximum correlation intensity, as in the original method. Simulated results are presented for a two-class problem, as well as experimental results obtained in a VanderLugt correlator. The filters produce the desired correlation response in both cases.
\end{abstract}

Keywords: Synthetic Discriminant Function, Liquid Crystal Devices, correlation

\section{INTRODUCTION}

Filter design is an important field of research in optical pattern recognition. The most frequently used filters, when distortion invariance is desired, are the Synthetic Discriminant Function ones. ${ }^{1}$ Kumar ${ }^{2}$ did a summary of some of the most representative correlation filters, including SDF. This type of filter is constructed from a set of training images with a correlation response fixed for all the images that compound the filter. The distortion information included by the filter depends on the images used in its generation. SDF filters also allow inter-classes discrimination, because the fixed response can be rejection.

An algorithm for the generation of correlation filters has to take into account the characteristics of the Spatial Light Modulator (SLM) where it is displayed in the correlator. These modulators can only accommodate a small fraction of the complex plane, so an adaptation process is necessary. The MEDOF filters ${ }^{3}$ include these considerations for single-image filters, optimizing different metrics in the filter design, such as the relation between signal and noise in the correlation plane or the peak sharpness. The first attempts to include modulation in SDF considered phase-only modulation for the filter, without any optimization process, just using the phase of a composite filter generated in the conventional way. ${ }^{4}$ The results were not acceptable because the SDF constraints were no longer met, as Casasent and Rozzi showed. ${ }^{5}$ Since then, there have been other algorithms that have optimized SDF to phase-only modulation, such as Kallman's method, ${ }^{6}$ which is computationally intensive, or that of Jared and Ennis, ${ }^{7}$ an iterative procedure also known as the Relaxation Algorithm, which is the starting point from the method presented in this work. Montes et al $l^{8}$ presented an algorithm which achieved the generation of SDF taking into account an arbitrary modulation for the filter. The inclusion of scene modulation due of the SLM displaying is not mentioned in any of these works. Our aim is to include the modulation of both SLMs in the filter's design to implement it experimentally, generalizing the Jared-Ennis method to the complex plane, in order to achieve its convergence using an arbitrary modulation.

\footnotetext{
Correspondence to Encarnación Pleguezuelos: e-mail: encarni@fao.ub.es, telephone: +34 934021203
} 
In section 2 the Jared-Ennis method is briefly reviewed. In section 3 we explain the algorithm we use to generalize the Jared-Ennis method to the complex plane, and some aspects of the simulation are discussed. Some simulated results are shown in section 4 and in section 5 we present some experimental results using a VanderLugt correlator with two LCDs as SLM. Finally, in section 6, conclusions are discussed.

\section{THE JARED-ENNIS METHOD}

The method proposed in this work is based on the Jared-Ennis algorithm for the generation of SDF filters. These filters are constructed as an arbitrary function of a set of training images, fixing the central amplitude correlation between each image and the filter, as

$$
\left[t_{n}(x, y) \otimes h(x, y)\right]_{(0,0)}=c_{n},
$$

where $t_{n}(x, y)$ is one of the $\mathrm{N}$ images of the training set, $h(x, y)$ is the filter in the real space. The inclusion of more than one image allows the consideration of different distortions. In the composite filter, the type of SDF filter considered in the Jared-Ennis method, the filter is a linear combination of the images as

$$
h(x, y)=\sum_{n=0}^{N} a_{n} t_{n}(x, y),
$$

where $a_{n}$ is the coefficient of the $t_{n}(x, y)$ image. If we substitute equation 2 into equation 1 the result is a system of linear equations ( $\mathrm{N}$ equations, one for each image of the training set) that can be analytically solved. When an arbitrary modulation is considered, the equations have no analytical solution in general.

The Jared-Ennis method is an iterative procedure for solving the non-linear equations system for the coefficients $a_{n}$, considering no modulation for the images of the training set and phase-only modulation or binary phase-only modulation for the filter. The iterative method does not work under other modulations. ${ }^{7}$ The iteration for the coefficients is as follows:

$$
\begin{aligned}
& a_{n}^{i+1}=a_{n}^{i}+\beta\left(c_{n}-c_{0} \frac{m_{n}^{i}}{m_{0}^{i}}\right), \\
& m_{n}^{i}=\left[t_{n}(x, y) \otimes h^{i}(x, y)\right]_{(0,0)},
\end{aligned}
$$

where $i$ is the iteration number and $\beta$ is a parameter which Jared and Ennis fixed to 0.3. The convergence depends on this parameter. $m_{n}^{i}$ is the correlation between each image of the training set and the filter generated in the $i$ iteration. The initial conditions for the iteration are $a_{n}^{0}=c_{n}$. When the $m_{n}^{i}$ central correlation amplitudes are equal to the fixed ones $\left(c_{n}\right)$ the method has converged in the solution. This algorithm presents some advantages, such as its mathematical simplicity and its fast convergence in the solution. Because of these advantages, we considered this algorithm as a basis for our method. However, there is a need for its generalization to any modulation for the filter and the inclusion of the scene modulation. The method presented in this work deals with these problems, as shown in the following section. There have been previous works related to the generalization of the Jared-Ennis method for achieving the introduction of more than one image in the generation of MEDOF filters. ${ }^{9}$

\section{GENERALIZATION TO THE COMPLEX PLANE}

The method we present includes arbitrary modulation for the filter and the images considered in the filter construction. This is indispensable for the experimental verification of the method. The operative curves used in this work were obtained from the experimental characterization of two LCDs, ${ }^{10}$ which are shown in figure 1. We use a high contrast modulation for scenes and a phase-mostly one for the filter. However, the modulations considered can be different: our method can be used with any configuration.

The filter is generated in the Fourier space as follows:

$$
H(u, v)=\mathcal{M}_{f}\left[\mathcal{F}\left(\sum_{n=0}^{N-1} a_{n} \mathcal{M}_{i}\left[t_{n}(x, y)\right]\right)^{*}\right],
$$




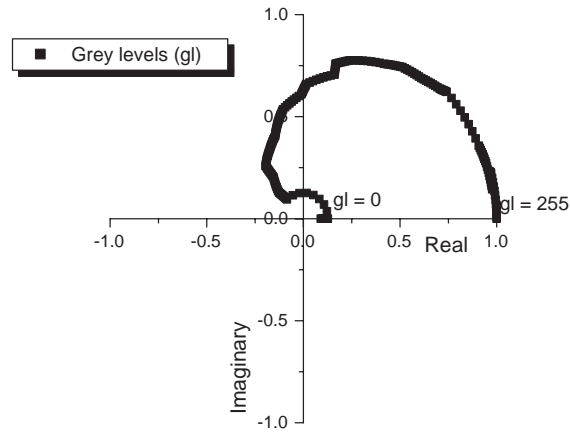

(a)

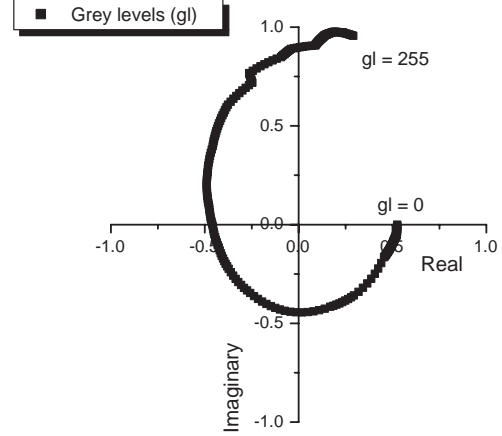

(b)

Figure 1. Operative curves considered in the filter generation: (a) modulation of the scene (high contrast configuration), (b) modulation of the filter (phase mostly configuration).

where $\mathcal{M}_{f}$ and $\mathcal{M}_{i}$ are respectively the modulation for the filter and the images. The linear combination of the modulated images is transformed into the Fourier space and then conjugated. Then it is adapted to the second operative curve. The coefficients of the linear combination are no more restricted to be real because the modulated images have amplitude and phase values, and so we allow them having complex values to add parameters to the problem. Therefore, the fixed correlations $c_{n}$ are complex valued. Each equation 1 (one for each image of the training set) is divided in its real and imaginary parts and we apply the formula 3 of the Jared-Ennis method to iterate the real and imaginary part of the coefficients, as shown in equations 6 and 7 .

$$
\begin{gathered}
\mathcal{R}\left(a_{n}^{i+1}\right)=\mathcal{R}\left(a_{n}^{i}\right)+\beta\left(\mathcal{R}\left(c_{n}\right)-\mathcal{R}\left(c_{0}\right) \frac{\mathcal{R}\left(m_{n}^{i}\right)}{\mathcal{R}\left(m_{0}^{i}\right)}\right), \\
\mathcal{I}\left(a_{n}^{i+1}\right)=\mathcal{I}\left(a_{n}^{i}\right)+\beta\left(\mathcal{I}\left(c_{n}\right)-\mathcal{I}\left(c_{0}\right) \frac{\mathcal{I}\left(m_{n}^{i}\right)}{\mathcal{I}\left(m_{0}^{i}\right)}\right),
\end{gathered}
$$

In order to verify the convergence of this generalization, we provide an example of a filter construction. Figure 2 shows the images used in the training set in this work. Class A is defined as the dolphin and its in-plane rotation and class B is the shark and its rotation. In figure 3 the evolution of the central correlation amplitude in the iteration is shown for the generation of a filter constructed from two class A and two class B images. The $\beta$ parameter has been fixed to 0.3 as in the original method and the initial coefficient values are chosen to be equal to the fixed correlations too. These correlation values are set to $c_{n}=\frac{(1,1)}{\sqrt{2}}$ for class $\mathrm{A}$ and $c_{n}=(0,0)$ for class B. We can see that the correlation converges in a few iterations (less than thirty) to the fixed values.

The method maintains the mathematical simplicity of the original and although the calculus time has increased because of the adaptation to the modulation, it is not critical. The time the algorithm takes for iteration is about one minute in a Pentium-IV computer using 36 images of $512 \times 512$ pixels.

\section{SIMULATED RESULTS}

We have carried out some simulations to test the performance of the filters generated using this method. First of all, a filter from 36 images belonging to class A, dolphins rotated $10^{\circ}$, has been constructed for the recognition of one class images. Figure 4 shows the central correlation amplitude obtained between each image and the filter. The error is close to zero (the mean value is 1 and the standard deviation is $7.5 \times 10^{-4}$ ). The filter gives the desired correlation responses for the images considered in its construction. 


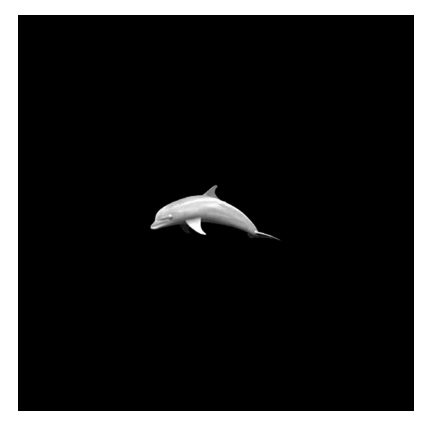

(a)

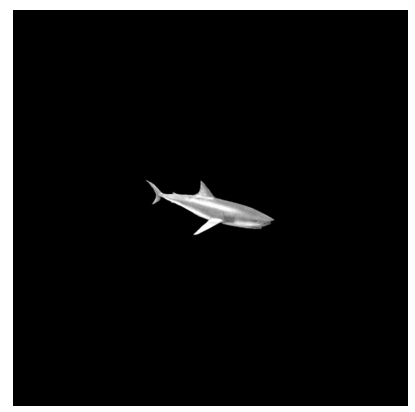

(b)

Figure 2. Images belonging to the training set: (a) class A, (b) class B.

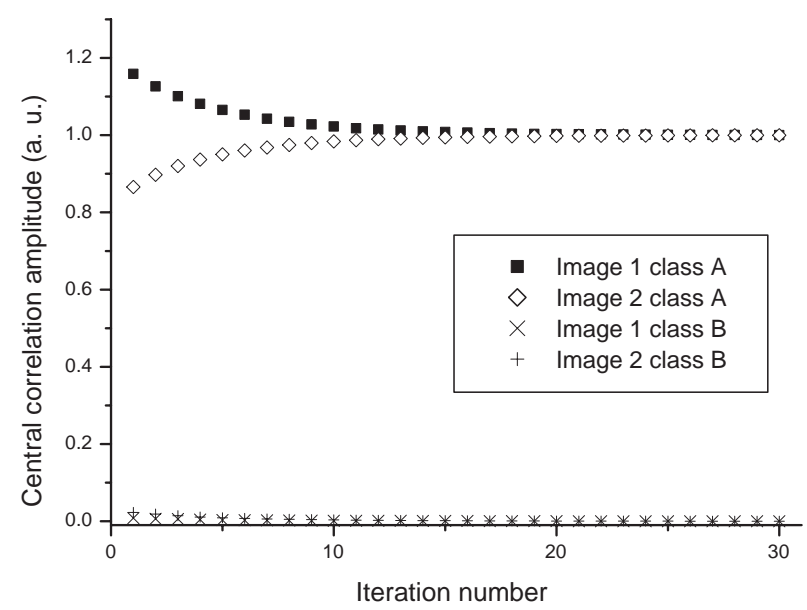

Figure 3. Evolution of the central correlation amplitude during the iteration in the generation with the generalized method of a filter that includes four images, with the detection of class A.

Figure 5(b) shows the three dimensional representation of the correlation plane simulated with a filter of 18 images belonging to class $\mathrm{A}$ and a scene containing a dolphin and a dolphin rotated $20^{\circ}$. The detection is clear for both targets and the same intensity is achieved.

To analyze the filter response to images other than that included in the filter construction, we have generated a filter from 18 images, all of them belonging to class A: dolphins rotated $20^{\circ}$. In figure 6 we show the central correlation amplitude between a filter and each of the 36 images considered in its generation (dolphins rotated each $10^{\circ}$ ). Responses for images included in the filter are those we expected. However, the correlation amplitude for the non-considered images is lower and so we could not conclude if there had been detection. Therefore, this filter cannot describe the target properly and lower angular separation has to be taken if we desire invariance to this distortion. An increase in images in the training set could solve this drawback.

We have used the Peak to Correlation Energy (PCE) as a parameter to study the influence of the number of images included in the filter construction on the correlation plane. PCE is defined as the relationship between the peak maximum value and the correlation plane energy, as follows: 


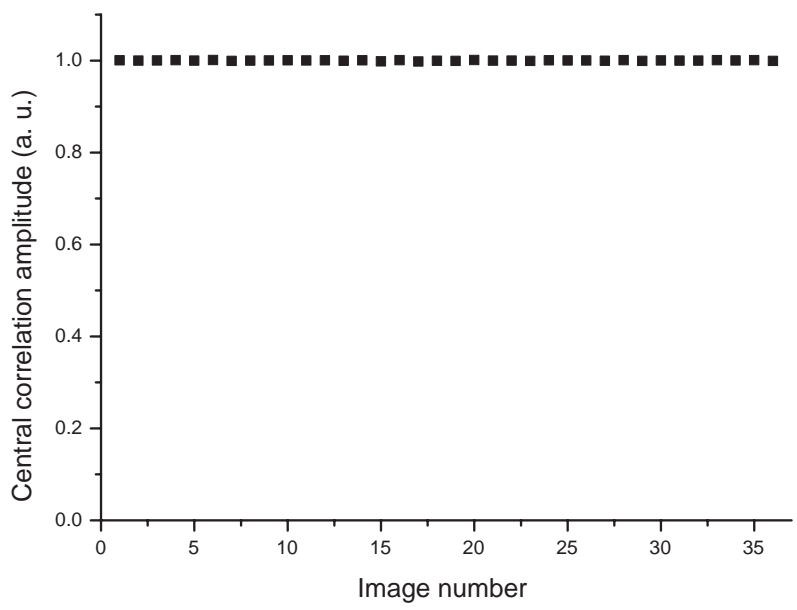

Figure 4. Central correlation amplitude with each of the 36 images belonging to class A and a filter generated from all of them.

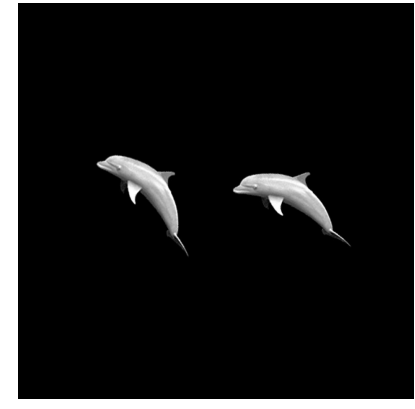

(a)

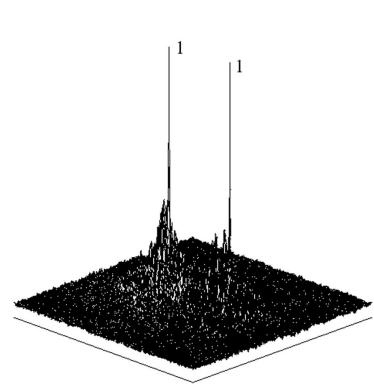

(b)

Figure 5. (a) Simulated correlation plane between the input scene (b) and a filter generated from 18 images belonging to class A.

$$
P C E=\frac{\|c(0,0)\|^{2}}{\iint\|c(x, y)\|^{2} d x d y} .
$$

In figure 7 we show the PCE in simulated correlation planes between the image in figure 2(a) and a filter that always detects it. The $\mathrm{x}$-axis is the number of images considered in the filter construction. We can see that the PCE value decreases as the number of images increases. This fact is a consequence of the lack of resolution, which is limited to the grey levels available in the LCD (256 levels). Therefore the number of images included in the filter is not unlimited, because its discrimination capabilities get worse when the number of images increases. As the number of images considered might be high if we want to describe an object, more than one filter might be used in the recognition process.

Next we discuss the two-class problem. The central correlation responses are the desired ones using a filter constructed from 36 images, half of each class, which detects class A (figure 8). As the algorithm only fixes the central correlation amplitude, the maximum for a correlation with a rejected image will be placed in another 


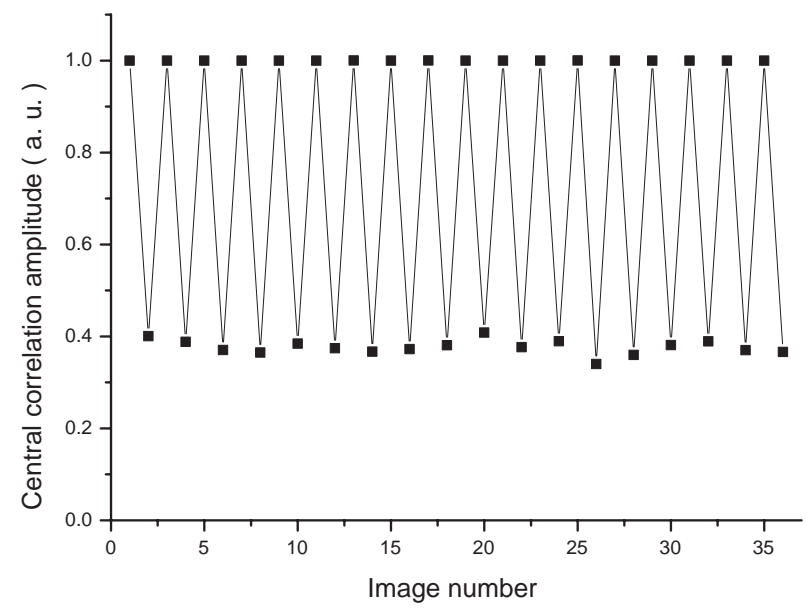

Figure 6. Central correlation amplitude with each of the 36 images belonging to class A and a filter generated from 18 of them.

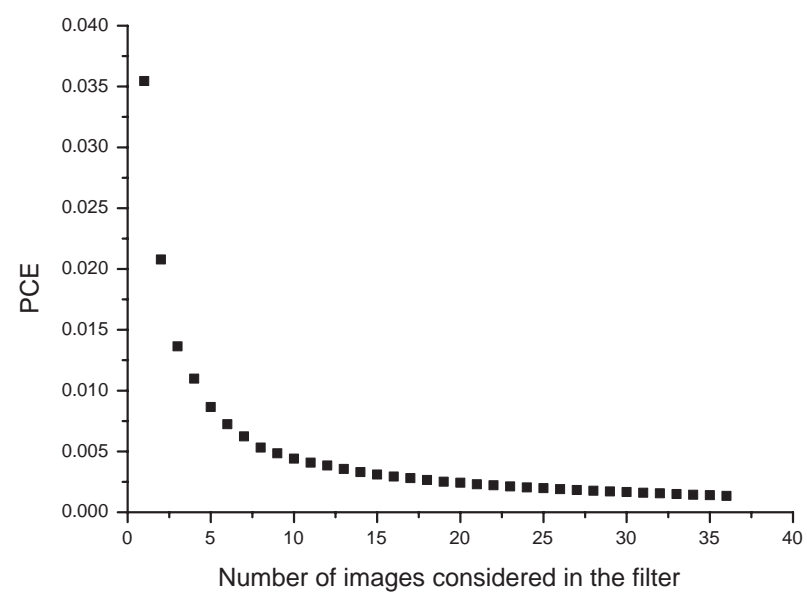

Figure 7. PCE calculated from the simulated correlation between an image and filters generated from a different number of images.

point of the correlation plane. This fact can lead to false detections, which is the SDF filters' main drawback. The simulation of the correlation between a filter and a scene with one image of each class is shown in figure 9. The filter in this case has been constructed from 10 images of class B, which are the target, and 10 of class A, whose correlation has been fixed to 0 , so the dolphins are the class to be rejected. As we can see, while the response for the target is the desired one, the maximum (not the central value) in the area corresponding to the dolphin is not 0 , although it is lower and the detection is clear. In the next section we will present some results obtained experimentally using the same modulation considered in the generation of the filter. 


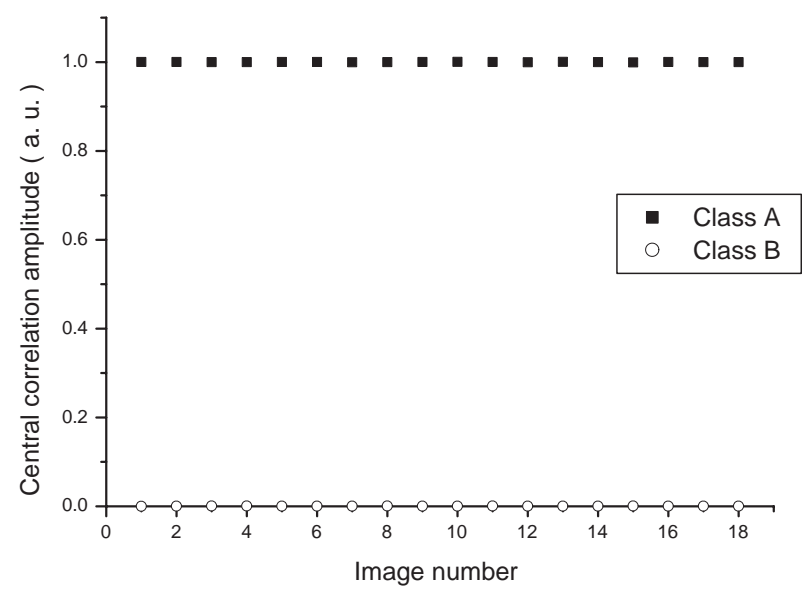

Figure 8. Central correlation amplitude with each of the 36 images included in the generation of the filter, 18 belonging to each class. The detected class is A.

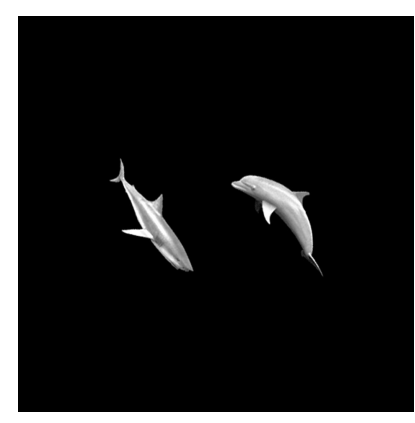

(a)

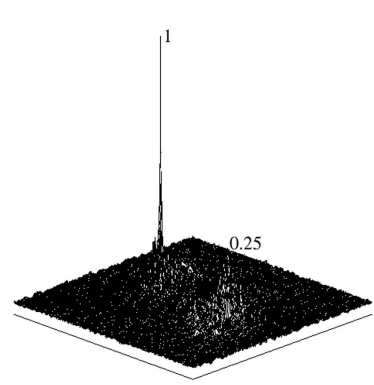

(b)

Figure 9. (a) Input scene (b) and simulated correlation plane between the scene and a filter generated from 20 images, 10 belonging to each class. The detected class is B.

\section{EXPERIMENTAL RESULTS}

The experimental setup used in this work is a convergent VanderLugt correlator with two LCDs removed from an EPSON EMP-3000 video projector. We use a He-Ne laser source of $632.8 \mathrm{~nm}$ and a CCD camera to capture the correlation plane. The LCDs work under the configurations shown in figure 1. As the filters generated with our method are optimized to both LCDs configurations, we can check the performance of the algorithm experimentally. In this section some experimental results are shown for a single class problem and a two-class problem, studying the influence of the number of images included in the training set.

The central correlation intensity captured with a filter constructed from 18 images and each of these images (figure 10) presents a standard deviation higher than in the simulated case (figure 4). The standard deviation value is 0.049 with a mean value of 0.88 . However we can conclude detection for all the images considered, as the peaks are higher than for a non-detected class. The main error sources may be misalignments of the correlator or the stability of the operative curves. ${ }^{11}$ In figure 11 there is the input scene (a) and the three dimensional representation of a correlation plane captured experimentally (b) with this scene and a filter generated from 18 images belonging to class $\mathrm{A}$, all of them detected. We can see that there is a slight difference between the two 
maximums but the result is good, although the correlation plane presents more noise than in a simulated case (see figure 5 , for instance).

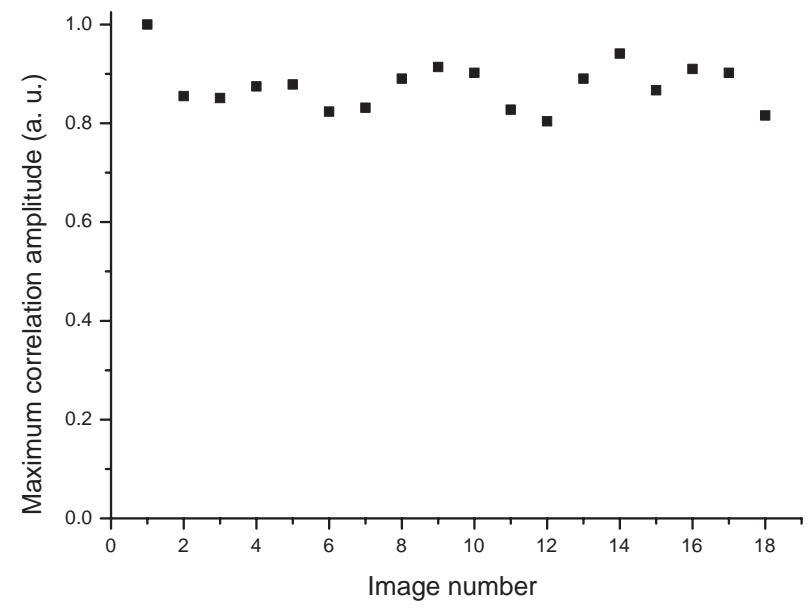

Figure 10. Central correlation amplitude with each of the 18 images included in the generation of the filter, belonging to class A, dolphins with a rotation of $20^{\circ}$.

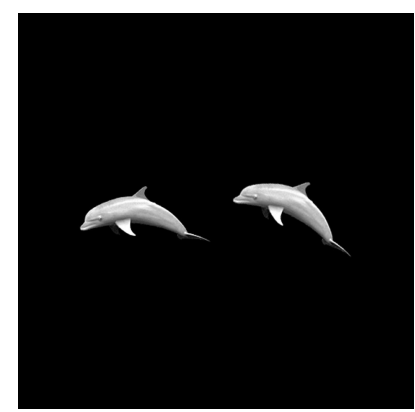

(a)

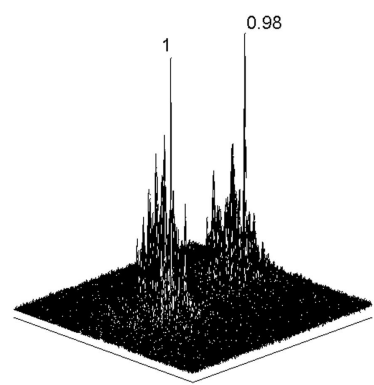

(b)

Figure 11. (a) Experimental correlation plane captured with the input scene (b) and a filter generated from 18 images belonging to class $\mathrm{A}$.

For a two-class problem we have chosen a scene (figure 9(a)) with a shark and a dolphin, and we have captured the correlation using two filters, constructed from 8 and 16 images, half of each class, shown in figures 12(a) and 12(b) respectively. The class that both filters detect is B. As we can see, the result is worse when the number of images in the filter increases, as shown before. For the correlation plane captured with 8 images the discrimination is clearer than in the 16-image case. In addition to this, the correlation plane is lower (the PCE is higher). To summarize, the experimental results are in agreement with the simulated ones, and the correct behavior of the algorithm has been proved. 


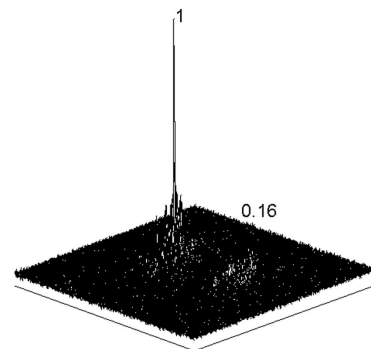

(a)

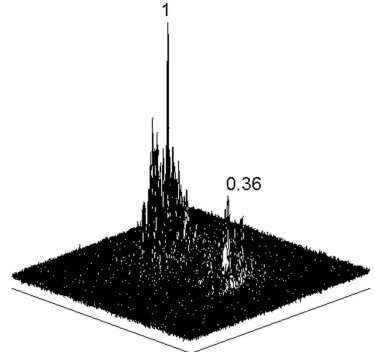

(b)

Figure 12. (a) Experimental correlation plane captured with the input scene in figure 9(b) and two different filters detecting class B: (a) generated from 8 images, 4 of each class and (b) generated from 16 images, 8 of each class.

\section{CONCLUSIONS}

In this work we have presented an algorithm for the design of SDF filters taking into account arbitrarily constrained devices to display images, both filter and scene, in a VanderLugt correlator. The algorithm is a generalization of the Jared-Ennis method to the complex plane which maintains its simplicity and its fast convergence to the solution. Simulated results are shown to illustrate the performance of the filters generated using this method. Besides the single class problem, the results obtained are those we expected for a two-class problem. Experimental results obtained in a convergent VanderLugt correlator have also been shown, presenting good agreement with simulated results.

\section{ACKNOWLEDGMENTS}

This paper has been partially funded by the CICYT (Comisión Interministerial de Ciencia y Tecnologia) through project DPI 2001-3365 and by the CIRIT (Comissió Interdepertamental de Recerca i Tecnologia) through project 2001SGR00079.

\section{REFERENCES}

1. C. F. Hester and D. Casasent, "Multivariant technique for multiclass pattern recognition," Applied Optics 19, pp. $1758-1761,1980$.

2. B. V. K. V. Kumar, "Tutorial survey of composite filter designs for optical correlators," Applied Optics 31, pp. 4773-4801, 1992.

3. R. D. Juday, "Optimal realizable filters and the minimum euclidean distance principle," Applied Optics 32, pp. 5100-5111, 1993.

4. J. L. Horner and P. D. Gianino, "Applying the phase-only filter concept to the synthetic discriminant function correlation filter," Applied Optics 24(6), pp. 851-855, 1985.

5. D. Casasent and W. A. Rozzi, "Computer-generated and phase-only synthetic discriminant function filters," Applied Optics 25(20), pp. 3767-3772, 1986.

6. R. R. Kallman, "Direct construction of phase-only filters," Applied Optics 26(24), pp. 5200-5201, 1987.

7. D. A. Jared and D. J. Ennis, "Inclusion of filter modulation in synthetic-discriminant-function construction," Applied Optics 28, pp. 232-239, 1989.

8. M. Montes-Usategui, J. Campos, and I. Juvells, "Computation of arbitrarily constrained synthetic discriminant functions," Applied Optics 34(20), pp. 3904-3914, 1995.

9. D. W. Carlson and B. V. K. V. Kumar, "Synthetic discriminant functions for implementations on arbitrarily constrained devices," in Optical Information Processing Systems and Architectures, B. Javidi, ed., Proc. SPIE 1772, pp. 10-20, 1992. 
10. E. Martín-Badosa, A. Carnicer, I. Juvells, and S. Vallmitjana, "Complex modulation characterization of liquid crystal devices by interferometric data correlation," Meas. Sci. Technol. 8, pp. 764-772, 1997.

11. M. Montes-Usategui, S. E. Monroe, and R. D. Juday, "Automated self-alignment procedure for optical correlators," Opt. Eng. 36, pp. 1782-1791, 1997. 